\title{
Does matching matter? Examining matches and mismatches between patient attributes and therapy techniques in alcoholism treatment
}

\author{
Mitchell P. Karno' \& Richard Longabaugh ${ }^{2}$ \\ Integrated Substance Abuse Programs, University of California, Los Angeles, CA, USA' and Center for Alcohol and Addiction Studies, Brown University, Providence, \\ $\mathrm{RI}, \mathrm{USA}^{2}$
}

\begin{abstract}
Aims This study assessed the predictive validity of the level of matching and mismatching between patients' personal attributes and aspects of outpatient psychotherapy they received. Design and participants On the basis of patientby-treatment interactions observed for this sample in previous research, patients with alcohol abuse or dependence $(n=137)$ were designated retrospectively as being matched, unmatched or mismatched on each of four patient and treatment variable pairings. These pairings included (1) patient depressive symptoms and therapy emotion focus, (2) patient trait anger and therapy confrontation, (3) patient interpersonal reactance and therapy confrontation and (4) patient interpersonal reactance and therapy structure. Measurements Analyses of variance and logistic regression were used to assess the individual and additive effects of being matched and mismatched on the percentage of abstinent days (PDA) and recovery status in the year after treatment. Findings Being mismatched on any of the four patienttreatment pairings was a significant predictor of more frequent alcohol use post-treatment. Being matched on only two pairings predicted less frequent alcohol use, namely matches on therapy emotion focus with patient depressive symptoms and therapy structure with patient reactance. Matches appeared to optimize otherwise good outcomes, while mismatches had larger effect sizes and tended to predict relatively poor outcomes. The data supported the presence of an additive effect for mismatches on post-treatment PDA. The group with the most mismatches fared considerably worse than a group with fewer mismatches. Several matches and mismatches also predicted recovery status, with some support found for additive effects. Conclusions Mismatches between patient attributes and treatment appear to have serious consequences, and this effect is magnified with multiple mismatches. Matches, on the other hand, while beneficial, may not be necessary to achieve good outcomes.
\end{abstract}

Keywords Alcoholism, matching, recovery, treatment.

Correspondence to: Mitchell P. Karno, Integrated Substance Abuse Programs, UCLA-ISAP, 11075 Santa Monica Blvd, Suite 200, Los Angeles, CA 90025, USA. E-mail: karno@ucla.edu

Submitted 10 February 2006; initial review completed 6 July 2006; final version accepted 14 November 2006

\section{INTRODUCTION}

The over-riding challenge of alcohol treatment research is to increase the effectiveness of alcohol interventions. In recent years three strategies have been prominent. One is the creation of new treatments that may be used to augment existing treatments. Pharmacotherapies such as naltrexone and acamprosate are innovations that have demonstrated potential [1-3], although not consistent incremental efficacy over placebo and psychosocial treatments $[4,5]$.
A second strategy is to combine elements of what is believed to be the most effective existing treatment modalities into single treatment protocols. A recent example is the Combine Behavioral Intervention in Project Combine [6,7] that integrates a motivational interviewing style with modules selected from cognitive behavioral therapy [8], the community reinforcement approach [9] and techniques to facilitate utilization of self-help groups such as Alcoholics Anonymous [10].

A third strategy has been to try to enhance outcomes through the identification of particular treatment 
strategies best suited to particular patients. There was keen interest in this so-called 'matching' paradigm prior to Project MATCH $[11,12]$. Indeed, 31 successful matching studies had reported improving the outcomes of patients with alcohol problems by pairing them with identifiable treatment settings and approaches [13]. The promising results from these studies led the National Institute on Alcohol Abuse and Alcoholism (NIAAA) to initiate the largest multi-site, multi-center randomized clinical trial of psychosocial treatments ever undertaken. Three popular and distinctive treatment modalities, cognitive behavioral therapy (CBT), motivational enhancement therapy (MET) and 12-Step facilitation therapy (TSF) were compared in their effectiveness for patients matched on 21 attributes. The results from this methodologically rigorous landmark study were disappointing. Of 35 a priori matching hypotheses, only four were supported and each accounted for only a small fraction of the variance in outcome $[11,12]$.

The impact of the primarily negative results of this model study has been to bring matching research to a virtual halt. Clearly, the matching paradigm has been generally discarded as a promising strategy for enhancing patient outcomes. However, as discussed in one of the Project MATCH papers [12], that study tested only a limited domain among treatment variables when it contrasted three individual treatment modalities (CBT, MET and TSF) with one another. Other variables in therapy were not examined for their matching potential. Nevertheless, the prevailing view among alcohol treatment researchers is that the matching paradigm has outlived its usefulness.

Positive findings from more recent research on patient-treatment interactions suggest that it is premature to declare that matching is dead [14-17]. This research on behavioral treatment for alcoholism examined treatment effectiveness as a function of interactions between therapist behaviors and patient attributes. The work represented a paradigmatic shift away from studying discrete treatment approaches in favor of studying the role of specific therapist behaviors. Through the use of observational coding of alcohol treatment sessions sampled from a clinical research unit of Project MATCH, the method yielded promising results about four patient-treatment interactions. Those interactions were between patients' depressive symptoms and the therapists' focus on emotional material during treatment sessions [14], between patients' level of trait anger and therapists' use of confrontation [15,16], between patients' level of trait reactance (i.e. the extent to which an individual generally resists being influenced by others) and therapists' use of confrontation [16,17] and between patients' level of trait reactance and therapists' use of structure [16,17].
The identification of these interaction effects was important because it provided evidence that the effectiveness of behavioral treatment for alcoholism could be enhanced by systematically tailoring therapy interventions to particular patients. Overall, the findings indicated that (1) a focus on emotional material in therapy was associated with more frequent alcohol consumption among patients high in depressive symptoms [14], (2) therapist use of confrontation was associated with more frequent alcohol consumption among patients at medium and high levels of trait anger while it was associated with less frequent alcohol consumption among patients low in anger [15,16], (3) confrontation was associated with more frequent alcohol consumption among patients high in interpersonal reactance [16,17] and (4) therapy structure was associated with more frequent alcohol consumption among patients at medium or high levels of reactance [16,17].

Although the mechanisms underlying these effects have yet to be confirmed empirically, explanations have been suggested. It is thought that patients high in depressive symptoms are in a high state of emotional arousal and that emotion-focused interventions maintain that arousal and thereby increase the likelihood of future alcohol use [14]. People with high trait anger often feel they are treated unfairly and feel frustrated as a result [18], thus confrontation may elicit frustration that impacts alcohol use negatively [16]. People low in anger tend to use the defenses of denial and repression excessively [18] and these individuals may benefit from therapist confrontation because it challenges them to address issues that impact their alcohol use [16]. Finally, individuals high in reactance tend to resist being controlled by others, hence for these patients treatment adherence may drop when a therapist uses confrontation to address topics the client does not want to discuss or uses structure to control the direction of the session [16].

This recent research did well to identify patient groups for whom a particular therapist intervention was associated positively or negatively with post-treatment alcohol use. Yet an important limitation of the work was that it did not examine the extent to which individuals who received optimal interventions (e.g. highly reactant patients whose therapists used no confrontation) were better off compared to other patients. Similarly, those earlier findings also did not examine the extent to which individuals who received contraindicated interventions (e.g. highly reactant patients whose therapists were confrontational) were worse off compared to other patients. Such information is necessary both to estimate the real importance of these effects in a treatment setting and to provide empirically based recommendations about what interventions therapists should and should not use during treatment. 
In an effort to increase the clinical value of the previous findings, the current study conducts new analyses of the earlier data set to examine the four patient-treatment interactions in terms of optimal (i.e. 'matched') and contraindicated (i.e. 'mismatched') interventions. The first aim of this study is to assess the impact of patients being matched or mismatched on the therapy interventions of emotion-focus, confrontation and structure. The presence of multiple interaction effects also allows for a rare opportunity to assess if multiple matches or mismatches are associated with larger changes in alcohol use. The second aim of the study is therefore to determine if being matched or mismatched on multiple effects has an additive effect on outcome.

With regard to the first aim and based on previous findings, we hypothesize that less frequent alcohol use after treatment and a greater likelihood of recovery will be associated with the following patient-treatment matches from the four interaction effects: (1) low emotion-focus therapy for patients high in depressive symptoms, (2) low confrontation therapy for patients at medium or high trait anger and high confrontation therapy for patients at low trait anger, (3) low confrontation therapy for patients high in reactance and (4) low structure therapy for patients at medium or high reactance. Conversely, we hypothesize that more frequent alcohol use after treatment and a lower likelihood of recovery will be associated with the following mismatches from the four interaction effects: (1) high emotion-focus therapy for patients high in depressive symptoms, (2) high confrontation therapy for patients at medium or high trait anger and low confrontation therapy for patients at low trait anger, (3) high confrontation therapy for patients high in reactance and (4) high structure therapy for patients at medium or high reactance.

With regard to the second aim, we hypothesize that an increasing number of matches will be associated with an incremental increase in abstinent days and the likelihood of recovery, and that an increasing number of mismatches will be associated with an incremental decrease in abstinent days and the likelihood of recovery.

\section{METHOD}

\section{Participants}

The sample $(n=137)$ all received treatment through the Providence Clinical Research Unit (CRU) of Project MATCH. Based on participants' informed consent to participate in Project MATCH, the Brown University Institutional Review Board (IRB) approved and oversaw the present study. All patients met criteria for a Diagnostic and Statistical Manual version III-revised (DSM-IIIR) diagnosis of alcohol abuse or alcohol dependence and participated in either inpatient or day hospital treatment for their alcohol use prior to enrollment in Project MATCH. The sample was $31 \%$ female. The average age was 44.7 years, ( $S D=13.2$ years), with 96\% Caucasian and $4 \%$ African American. This sample represented $81.5 \%$ of the total participants $(n=168)$ who enrolled in the Providence CRU of Project MATCH. Thirty-one cases were excluded because of missing data, including having no recorded treatment session available $(n=19)$, no information concerning alcohol use after treatment $(n=8)$ or missing a measure of a key baseline patient attribute $(n=4)$.

\section{Treatments in Project MATCH}

The three psychosocial treatments implemented in Project MATCH were cognitive-behavioral coping skills therapy (CBT), motivational enhancement therapy (MET) and 12-Step facilitation (TSF). All treatments were delivered in an individual format and were time-limited for 12 weeks. At the Providence CRU of Project MATCH a total of nine therapists delivered treatment and therapists were nested within treatment modality.

\section{Measurement of patient attributes}

The three patient attributes of depressive symptoms, trait anger and interpersonal reactance (i.e. the extent to which an individual resists being influenced by others) were measured by the Beck Depression Inventory (BDI) [19], the Trait Anger Scale (TAS) [18] and the Reactance subscale of the Systematic Treatment Selection Clinician Rating Form [20], respectively. The BDI and TAS are selfreport instruments and were administered to participants prior to treatment. The Reactance scale is an observerbased instrument that was applied to the first treatment session. The BDI is a 21-item scale with total scores ranging from 0 to 63 , the TAS is a 10-item scale with total scores ranging from 10 to 40 and the Reactance scale has 25 true or false items with a total score ranging from 0 to 25.

Patients were categorized into low, medium and high groups on each measure. On the BDI, the low, medium and high depressive symptom groups had scores at or below $9(n=73)$, between 10 and $18(n=39)$ and 19 or above $(n=25)$, respectively. Based on adult psychiatric norms, these groups reflected minimal, mild-moderate and moderate-severe depressive symptoms, respectively [19]. On the TAS the low, medium and high groups had scores at or below 14 (below 25th percentile of normal adult reference group, $n=12$ ), between 15 and 21 (between 25th and 75th percentiles of normal adult reference group, $n=77$ ) and 22 or above (above 75 th percentile of normal adult reference group, $n=48$ ), 
respectively [18]. For the Reactance scale normative data are not available. In the absence of such norms, the low, medium and high groups were designated as the lower third (mean $\pm \mathrm{SD}=2.31 \pm 0.94), n=43)$, middle third (mean $\pm \mathrm{SD}=5.54 \pm 1.09, n=46$ ) and upper third (mean $\pm \mathrm{SD}=11.45 \pm 3.20, n=48$ ) of the sample distribution.

\section{Measurement of therapy interventions}

The therapy interventions of emotion-focus, confrontation and structure were measured via the Therapy Process Rating Scale (TPRS) [21]. The TPRS is an observer-based measure. Each subscale contains fivepoint Likert-style items with a possible average score that ranges from 1 to 5 points. Two independent observers rated all items after viewing a segment of treatment on videotape that ranged from 15 to 25 minutes in length. Exact segment lengths were assigned for each session to ensure that the entire session was rated (e.g. a 60-minute session was divided into three equal-length segments of 20 minutes each). A total of four sessions (sessions 1, 2, 3 and the final session attended) were rated for each participant to assess interventions both early and late in treatment. In the event that participants did not attend four sessions, all attended sessions were rated. The independent ratings across all rated sessions were averaged to provide an overall measure of the intervention. (For more details on the therapy rating procedures see Karno \& Longabaugh [14]).

The emotion-focus subscale includes four items that measure the extent to which a therapist moves a patient towards or away from emotionally charged topics. The subscale had very good internal consistency (Cronbach's $\alpha=0.90$ ) and very good inter-rater reliability $($ ICC $=0.89)$ [14]. The confrontation subscale includes three items that measure therapist challenging of patient defenses and assigning meaning to the patient's experiences and resistance to treatment. The structure subscale includes three items that measure the therapist's use of closed-ended questions, providing information and initiating topics. In the TPRS, the confrontation and structure subscales were combined initially as a measure of directiveness and had good inter-rater reliability $(\mathrm{ICC}=0.75)$. Subsequent research indicated that confrontation and structure were separate factors, each with good internal consistency as evidenced by factor loadings $>0.7$ [16].

For each patient, the therapy interventions they received were categorized into low, medium and high groups. Normative data are not available for the TPRS, hence the categories were obtained by dividing the sample into three roughly equal-sized groups. The low, medium and high groups for emotion-focus were designated as the lower third (mean $\pm \mathrm{SD}=2.99 \pm 0.08$, $n=46)$, middle third (mean $\pm \mathrm{SD}=3.1 \pm 0.03, n=45$ ) and upper third (mean $\pm \mathrm{SD}=3.36 \pm 0.11, n=46)$ of the sample distribution. The low, medium and high groups for confrontation were designated as the lower third (mean $\pm \mathrm{SD}=1.34 \pm 0.12, n=46)$, middle third (mean $\pm \mathrm{SD}=1.67 \pm 0.07, n=45)$ and upper third (mean $\pm \mathrm{SD}=1.97 \pm 0.17, n=46$ ) of the sample distribution. The low, medium and high groups for structure were designated as the lower third (mean $\pm \mathrm{SD}=2.82 \pm 0.17, \quad n=46), \quad$ middle third (mean $\pm \mathrm{SD}=3.24 \pm 0.10, n=45)$ and upper third (mean $\pm \mathrm{SD}=3.62 \pm 0.16, n=46)$ of the sample distribution.

\section{Assessing independence of patient and therapist variables}

Given the retrospective nature of the study, it was important to assess the degree to which the patient and therapist variables potentially influenced one another. Correlations between the BDI and emotion focus, between the TAS and confrontation, between reactance and confrontation and between reactance and structure were $r=0.11, r=0.025, r=0.035$ and $r=-0.09$, respectively. These low levels of association between the patient and therapist variables support their independence.

\section{Designating matches and mismatches}

Patients were categorized as matched, mismatched or unmatched to the therapy interventions based on empirical results from prior research on each attribute-bytreatment interaction. With regard to the interaction between depressive symptoms and the emotion focus of therapy, evidence suggested that only for patients high in depressive symptoms does the emotion focus of therapy impact future alcohol use [14]. The direction of that effect was such that among depressed patients more focus on emotional material was associated with worse outcomes. Thus for the present study, patients in the high depressive symptoms group were classified as matched if they received therapy with a low emotion focus $(n=10)$ and as mismatched if they received therapy with a high emotion focus $(n=9)$. All other patients were classified as unmatched on this effect $(n=118)$.

For the interaction between patient anger and therapist confrontation, prior research indicated that confrontation had a positive effect for individuals low in anger while it had a negative effect for individuals at medium and high anger [16]. To represent this pattern of results in the present study, patients were classified as matched if they were medium or high in anger and had low confrontation $(n=44)$ or if they were low in anger and had high confrontation $(n=6)$ (total matched group for this effect 
had $n=50$ ). Patients were classified as mismatched if they were medium or high in anger and had high confrontation $(n=40)$ or if they were low in anger and had low confrontation $(n=2)$ (total mismatched group for this effect had $n=42)$. The remaining patients were classified as unmatched $(n=45)$.

For the interaction between patient reactance and therapist confrontation, prior research indicated that confrontation had a detrimental effect for patients high in reactance but not for patients at lower levels of reactance [16]. For the present study, patients high in reactance who had low confrontation were therefore classified as matched $(n=16)$ and patients high in reactance who had high confrontation were classified as mismatched $(n=15)$. The remaining patients were classified as unmatched $(n=106)$.

Finally, prior research on the interaction between patient reactance and therapist structure suggested that structure was contraindicated for patients at a medium or high level of reactance [16]. Accordingly, in the present study patients at medium or high reactance in the low structure therapy group were classified as matched $(n=38)$ and patients at medium or high reactance in the high structure therapy group were classified as mismatched $(n=28)$. The remaining patients were classified as unmatched $(n=71)$.

\section{Dependent variable}

Frequency of alcohol use

Alcohol use frequency was assessed as the percentage days abstinent (PDA) from alcohol during the first year after treatment. PDA for each 90-day period following treatment was measured in Project MATCH via the Form 90 interview [22]. The average PDA across four consecutive Form 90 interviews was used as the measure of PDA over the entire year. PDA was negatively skewed and an arcsine transformation was conducted to approximate a normal distribution.

\section{Recovery status}

On the basis of recent work by Cisler et al. [23], in applying clinical significance methodology to alcoholism treatment trials, patients were classified as 'recovered' $(n=71)$ if they demonstrated reliable improvement in abstinent days from pretreatment to the post-treatment follow-up period and were also 'functional'. The criterion for reliable improvement was having a reliable change index $[24] \geq 1.96$. The criterion for being functional was being abstinent more than $95 \%$ of the days during the year after treatment. This functional level of abstinence was calculated to be two standard deviations above the average baseline percentage of abstinent days for the whole sample. Patients who were either not reliably improved or functional were classified as 'not recovered' $(n=66)$.

\section{Data analysis}

Individual effects of matches and mismatches

Analyses examined the relationship of the four match and mismatch effects on the two dependent variables of PDA and recovery status. Analyses of variance were used to test the hypotheses that being matched or mismatched predicted less and more frequent alcohol use, respectively. Logistic regression analyses were used to test the hypotheses that being matched or mismatched predicted recovery status. Each effect was dummy-coded as matched, mismatched or unmatched. The contrasts of interest from each analysis compared the matched versus unmatched groups and the mismatched versus unmatched groups. These contrasts indicated the beneficial or detrimental effects of matches and mismatches, respectively, in relation to a neutral unmatched group. Contrasts between the matched and mismatched groups were not of interest because we cannot know if such differences are attributable to a match, a mismatch, or some combination of both. Two analyses were conducted for each effect, one predicting PDA and the second predicting recovery status. Given the directional nature of the research questions, all tests were one-tailed with critical $\alpha=0.05$.

Control variables included baseline PDA and treatment assignment. Additionally, we were aware that some of the matched and mismatched groups were made up of patients at only high levels of depressive symptoms or reactance. To minimize the chance that these patient variables were confounds (e.g. an observed effect might be attributed to differences in depressive symptoms between groups instead of to a patient-treatment match), depressive symptoms and reactance were entered into their respective models as continuous covariates. The variable of patient anger was not a potential confound because the matched and mismatched groups each included patients at high and low anger levels. Anger was therefore not included in the models as a covariate.

\section{Additive effect of matches and mismatches}

Two analyses of variance were conducted, one analysis to test the hypothesis that more matches predicted less frequent alcohol use and one analysis to test the hypothesis that more mismatches predicted more frequent alcohol use. Two logistic regression analyses were conducted, one analysis to test the hypothesis that more matches predicted a greater likelihood of recovery and one analysis to test the hypothesis that more mismatches predicted a 
Table 1 Effects of patient-treatment matches on percentage of days abstinent (PDA) during the 12-month post-treatment period $(n=137)$.

\begin{tabular}{|c|c|c|c|c|}
\hline \multirow[b]{2}{*}{ Patient-treatment matches } & \multirow[b]{2}{*}{$t$} & \multirow[b]{2}{*}{$\varepsilon^{2}$} & \multicolumn{2}{|c|}{$\begin{array}{l}\text { PDA } \\
\text { Untransformed mean (SD) }\end{array}$} \\
\hline & & & Matched & Unmatched \\
\hline Patient depressive symptoms and therapy emotion focus & $1.67^{*}$ & 0.021 & $98.15(3.99)$ & $86.99(24.51)$ \\
\hline Patient trait anger and therapy confrontation & 1.48 & 0.016 & $93.83(13.28)$ & $86.21(26.16)$ \\
\hline Patient interpersonal reactance and therapy confrontation & 0.13 & $<0.001$ & $86.89(20.11)$ & $88.72(23.18)$ \\
\hline Patient interpersonal reactance and therapy structure & $2.19^{*}$ & 0.036 & $89.99(21.43)^{\mathrm{a}}$ & $88.55(23.60)$ \\
\hline
\end{tabular}

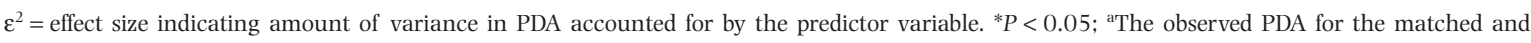
unmatched groups appear similar, yet after accounting for average levels of reactance and baseline PDA the estimated mean PDA for the matched group was $\mathrm{PDA}=95.34$ and for the unmatched group was $\mathrm{PDA}=84.89$.

lower likelihood of recovery. The number of matches and mismatches were considered to be ordinal variables, and there was no a priori assumption of a linear relationship between each of these variables and PDA. Hence the variables were entered into the models as categorical effects. Analyses were unweighted such that each effect was given equal weight without regard for its observed effect size in the earlier analyses. This strategy was selected in order to enhance the generalizability of the results by not overfitting the analyses to the current sample. Each analysis included those participants who were matched or mismatched on at least one effect. Consistent with the other analyses, control variables included baseline PDA, treatment assignment, depressive symptoms and reactance. Because anger was not a potential confound, it was not included in the models as a covariate. Tests were onetailed with critical $\alpha=0.05$.

\section{RESULTS}

Omnibus tests of patient-treatment matching effects

Prior to testing specific contrasts between the matched and unmatched groups and between the mismatched and unmatched groups, omnibus F-tests were conducted to determine if significant differences in PDA were observed across the groups in each of the four patient-treatment matching effects being studied. The results indicated a significant overall effect on PDA by the level of match (i.e. matched, unmatched or mismatched) for all the effects, including patient depressive symptoms and the emotion focus of therapy $\left[F_{(2,130)}=8.16\right.$, mean square error $(\mathrm{MSE})=0.13, \quad P<0.001]$, patient anger and therapist confrontation $\left(F_{(2,131)}=5.83, \quad \mathrm{MSE}=0.79\right.$, $P<0.01)$, patient reactance and therapist confrontation $\left(F_{(2,130)}=5.11, \mathrm{MSE}=0.13, P<0.01\right)$ and patient reactance and therapy structure $\left(F_{(2,130)}=6.26, \mathrm{MSE}=0.13\right.$, $P<0.01)$. In light of these omnibus effects we proceeded to test the specific contrasts of interest.

\section{On which effects did being matched matter?}

Results supported a significant positive effect on PDA of being matched on two of the four effects examined. These two effects were the match of therapy emotion focus with patient depressive symptoms and therapy structure with patient reactance. The effect of being matched on therapy confrontation with patient anger was not detected reliably. The effect of being matched on therapy confrontation with patient reactance was not a significant predictor of PDA. The effect sizes for the significant effects were in the medium range. The largest effect was the match of therapy emotion focus with patient depressive symptoms (Cohen's $d=0.55$ ), followed by the match of therapy structure with patient reactance (Cohen's $d=0.44$ ). These results are shown in Table 1 .

Looked at in terms of untransformed alcohol use frequency data, patients whose depressive symptoms were matched to the emotion focus of therapy $(n=10)$ were, on average, abstinent on $98.15 \%$ of the days in the year after treatment while patients not matched on this effect ( $n=118$ ) were abstinent on $86.99 \%$ of the days. Patients whose reactance was matched to the level of therapy structure $(n=38)$ were abstinent, on average, $89.99 \%$ of the days compared to unmatched patients $(n=71)$ who were abstinent for $88.55 \%$ of the days. These observed PDA were similar, although after accounting for average levels of reactance and baseline PDA the estimated mean PDA for the matched and unmatched groups was $95.34 \%$ and $84.89 \%$, respectively. Clearly, participants across all groups faired well on average. The positive effect of a match appeared to optimize the outcome, while not being matched did not predict a low percentage of abstinent days.

With regard to recovery status, the same two matches were significant predictors. Patients who were matched on the effect of depressive symptoms and the emotion focus of therapy were more than five times more likely to be recovered than patients who were not matched on this 
Table 2 Effects of patient-treatment matches on likelihood of recovery during the 12-month post-treatment period $(n=137)$.

\begin{tabular}{lllll}
\hline & & & \% Recovered \\
\cline { 3 - 5 } Patient-treatment matches & & & Matched & Unmatched \\
\hline Patient depressive symptoms and therapy emotion focus & $5.51^{*}$ & $1.19-25.49$ & 80.00 & 52.54 \\
Patient trait anger and therapy confrontation & 1.33 & $0.65-2.73$ & 62.00 & 55.55 \\
Patient interpersonal reactance and therapy confrontation & 0.46 & $0.17-1.30$ & 37.50 & 58.49 \\
Patient interpersonal reactance and therapy structure & $5.71 \ddagger$ & $2.23-14.64$ & 71.05 & 49.30 \\
& & & & \\
\hline
\end{tabular}

OR = odds ratio, $\mathrm{CI}=$ confidence interval. ${ }^{*} P<0.05 ; \uparrow P<0.001$.

Table 3 Effects of patient-treatment mismatches on percentage of days abstinent (PDA) during the 12-month post-treatment period $(n=137)$.

\begin{tabular}{llllll}
\hline & & & \multicolumn{2}{c}{ PDA } \\
& & & & Untransformed mean (SD) \\
\cline { 2 - 6 } Patient-treatment mismatches & & & $\varepsilon^{2}$ & Mismatched & Unmatched \\
\hline Patient depressive symptoms and therapy emotion focus & $-2.95 \dagger$ & 0.063 & $51.19(39.70)$ & $86.99(24.51)$ \\
Patient trait anger and therapy confrontation & $-1.93^{*}$ & 0.028 & $74.68(34.33)$ & $86.21(26.16)$ \\
Patient interpersonal reactance and therapy confrontation & $-3.00 \dagger$ & 0.065 & $60.84(40.31)$ & $88.72(23.18)$ \\
Patient interpersonal reactance and therapy structure & $-1.80^{*}$ & 0.024 & $71.45(34.74)$ & $88.55(23.60)$
\end{tabular}

$\varepsilon^{2}=$ effect size indicating amount of variance in PDA accounted for by the predictor variable. ${ }^{*} P<0.05 ; \dagger P<0.01$.

effect. Patients who were matched on the effect of reactance and therapy structure were nearly six times more likely to be recovered than patients who were not matched on this effect. Being matched on the other effects was not a significant predictor of recovery status. These results are shown in Table 2.

\section{On which effects did being mismatched matter?}

As shown in Table 3, results supported a significant negative effect of being mismatched for all four of the effects examined. Predictors of more frequent alcohol use in the year following treatment included a mismatch of therapy emotion focus with patient depressive symptoms, therapy confrontation with patient anger, therapy confrontation with patient reactance and therapy structure with patient reactance. The magnitude of effect sizes for these effects ranged from large to medium. The mismatch of therapy emotion focus with patient depressive symptoms was a large effect (Cohen's $d=1.02$ ), followed by the mismatch of therapy confrontation with patient reactance (Cohen's $d=0.83$ ) and the mismatches of therapy confrontation with patient anger (Cohen's $d=0.42$ ) and therapy structure with patient reactance (Cohen's $d=0.40)$.

In terms of untransformed alcohol use frequency data, patients whose depressive symptoms were mismatched to the emotion focus of therapy $(n=9)$ were, on average, abstinent for only $51.19 \%$ of the days in the year after treatment while the unmatched patients on this effect ( $n=118$ ) were, on average, abstinent for $86.99 \%$ of the days. Patients whose anger was mismatched to the level of confrontation $(n=42)$ were abstinent for an average of $74.68 \%$ of the days post-treatment while the unmatched patients $(n=45)$ abstained for an average of $86.21 \%$ of the days. Patients whose reactance was mismatched to the level of therapy confrontation $(n=15)$ abstained on $60.84 \%$ of the days, on average, compared to the unmatched patients $(n=106)$ who, on average, abstained $88.72 \%$ of the days. Lastly, patients whose reactance was mismatched to the level of therapy structure $(n=28)$ abstained on $71.45 \%$ of the days compared to the unmatched patients $(n=71)$, who abstained on $88.55 \%$ of the post-treatment days on average.

These results indicated that being mismatched on each of these effects was associated with more frequent alcohol use. In contrast to the findings that showed being matched optimized otherwise good outcomes, these results suggested that the effects of being mismatched had a more profound impact on post-treatment alcohol use.

For recovery status, two of the four mismatches were significant predictors. Patients who were mismatched on the effect of anger and therapy confrontation were approximately one-half as likely to be recovered, and patients who were mismatched on the effect of reactance and therapy confrontation were approximately 
Table 4 Effects of patient-treatment mismatches on likelihood of recovery during the 12-month post-treatment period $(n=137)$.

\begin{tabular}{lllll}
\hline & & & \multicolumn{2}{c}{ \% Recovered } \\
\cline { 3 - 5 } Patient-treatment mismatches & & & Mismatched & Unmatched \\
\hline Patient depressive symptoms and therapy emotion focus & 0.17 & $0.02-1.20$ & 11.11 & 52.54 \\
Patient trait anger and therapy confrontation & $0.44^{*}$ & $0.21-0.95$ & 35.71 & 55.55 \\
Patient interpersonal reactance and therapy confrontation & $0.22^{*}$ & $0.06-0.74$ & 20.00 & 58.49 \\
Patient interpersonal reactance and therapy structure & 0.61 & $0.25-1.48$ & 32.14 & 49.30 \\
\hline
\end{tabular}

OR $=$ odds ratio, $\mathrm{CI}=$ confidence interval. ${ }^{*} P<0.05$.

one-quarter as likely to be recovered. Being mismatched on the effect of patient depressive symptoms and therapy emotion focus or on reactance and confrontation was not a significant predictor of recovery status. These results are displayed in Table 4.

\section{Is there an additive effect of matches?}

On the basis of the initial findings that only two of the four match effects were predictors of PDA and recovery status, we included in this analysis only those two effects that were significant predictors. Thus, patients could be matched on up to two effects. The distribution was such that patients had no matches $(n=93)$, one match $(n=40)$ or two matches $(n=4)$. Unfortunately, given that only four cases were matched on both the effects, we deemed that sample size too small for the purpose of conducting a statistical analysis. Descriptively, however, the average levels of PDA are suggestive of an effect in the expected direction. Mean \pm SD levels of post-treatment PDA for the no-matches, one-match and two-matches groups were $82.85 \pm 28.77, \quad 90.09 \pm 20.90$ and $99.67 \pm 0.66$, respectively. In terms of recovery status a similar trend was observed. The percentage of recovered individuals in the zero-, one- and two-match groups was $43.01 \%, 67.50 \%$ and $100 \%$, respectively.

\section{Is there an additive effect of mismatches?}

On the basis of the initial findings that all four mismatch effects were predictors of PDA, we included in this analysis all of the mismatch effects. Thus, patients could be mismatched on up to four effects. The distribution was such that patients had no mismatches $(n=80)$, one mismatch $(n=31)$, two mismatches $(n=17)$, three mismatches $(n=7)$ and four mismatches $(n=2)$. Given the relatively few participants who were mismatched on three or four effects, those two groups were combined into one group $(n=9)$. For the resulting four-level variable three contrasts were planned comparing post-treatment PDA between those groups with (1) no mismatches versus one mismatch, (2) one mismatch versus two mismatches and (3) two mismatches versus three or four mismatches.
Results indicated a significant overall effect comparing PDA across the mismatch groups (omnibus $F_{(3,128)}=8.08$, MSE $\left.=0.935, P<0.001\right)$. The planned contrasts showed that patients with no mismatches had higher PDA on average (91.81\%) than those with one mismatch $(83.56 \% ; \quad P<0.05)$. No difference was observed in PDA between patients with one and two mismatches $(P>0.40)$, yet patients with two mismatches had substantially higher PDA on average (82.63\%) than did patients with three or four mismatches $(40.87 \%$; $P<0.001)$. The observed effect was medium-to-large, with Cohen's $d=0.65$.

For the dependent variable of recovery status, two individual mismatches were significant predictors. Hence a test of additivity compared patients with zero, one or two mismatches on those effects. The distribution was such that patients had no mismatches $(n=95)$, one mismatch $(n=27)$ or two mismatches $(n=15)$. Two contrasts were planned comparing post-treatment PDA between those groups with (1) no mismatches and one mismatch and (2) one mismatch and two mismatches.

Individuals with one mismatch were marginally less likely to recover than those with no mismatches [OR, 95\% CI $=0.48$ (0.22-1.08), $P=0.07]$, while the likelihood of recovery for the one- and two-mismatch groups did not differ [OR, 95\% CI $=1.84(0.46-7.32), P>0.20$ ]. The largest difference, however, was between the groups with no mismatches $(58.9 \%$ recovered) and two mismatches (20\% recovered) [OR, 95\% CI $=3.7$ (1.13-12.69), $P<0.05]$.

\section{DISCUSSION}

Two of the four patient-treatment pairings under investigation emerged as the most consistent predictors of outcome. On the pairings of patient depressive symptoms with therapy emotion focus and patient reactance with therapy structure, being either matched or mismatched was associated with the frequency of alcohol use in the expected directions. Being matched on these pairings was also associated with a greater likelihood of being 
classified as recovered. These results indicated that when treating patients high in depressive symptoms, directing them away from emotionally charged material (i.e. a match) was associated with a beneficial treatment effect, and directing them toward emotionally charged material (i.e. a mismatch) was associated with a negative treatment effect. For patients at medium or high levels of interpersonal reactance, a low level of therapy structure (i.e. a match) was associated with a beneficial treatment effect while high structure (i.e. a mismatch) was associated with a poorer outcome.

The patient-treatment pairings of patient anger with therapist confrontation and of patient reactance with therapist confrontation were predictors of outcome only when mismatches occurred. Worse outcomes on PDA and recovery status were observed for patients who were low in anger and mismatched with therapy low in confrontation, and for patients who were medium or high in anger and mismatched with therapy higher in confrontation. Similarly, worse outcomes on PDA and recovery status were observed for patients who were high in reactance and were mismatched with therapy high in confrontation. Matches on these effects did not predict either frequency of alcohol use or recovery status.

With regard to the additive effects of matches and mismatches, the data supported an additive effect of mismatches on the frequency of abstinent days and, to a lesser extent, recovery status. Patients with three or four mismatches drank more days, on average, during the year after treatment than patients with one or two mismatches. Further, patients with two mismatches were less likely to be recovered than patients with no mismatches. The limited cell sizes precluded formal tests of the additive effects of matches. Further study with larger sample sizes are needed to test those effects.

Importantly, the patient-treatment mismatch effects demonstrated consistently larger effect sizes compared to the match effects. These results suggest that mismatches played a larger role overall in predicting outcome than did matches. Interventions that were matched to a particular patient were beneficial, yet the matches did not appear to be a necessary condition for a good treatment outcome. Mismatches, on the other hand, were associated with a much greater frequency of post-treatment alcohol use compared to patients without mismatches.

The current study has several strengths and limitations. Among the notable strengths is the use of discrete clinical categories to classify patient-treatment pairs as matches or mismatches. The specific cut-off scores used to categorize the level of patient depressive symptoms, trait anger and reactance can be used in future research and can be incorporated into prospective treatment planning in clinical practice settings. The examination of differential and additive effects of potential matches and mismatches demonstrates that mismatching may be related more to treatment effectiveness than is matching. Finally, the inclusion of recovery status as a clinically significant outcome measure provides a vital test of the importance of these effects.

Limitations of the study include smaller-than-ideal cell sizes in the some of the analyses, the use of data from a single clinical research unit of Project MATCH and the absence of a prospective study to test the effects. Because this research was based on secondary analysis of the psychotherapy treatments in Project MATCH, matches and mismatches could not be manipulated. Hence the matches and mismatches under investigation occurred naturally in the course of treatment. The result was that most patients were neither matched nor mismatched to their therapy interventions. Consequently, the groups of matched and mismatched patients were small in some instances. While a retrospective analysis also cannot control to what extent therapist behaviors are influenced by patient behaviors during treatment, the correlations between the patient and therapist variables in the current study were very low.

The use of data from a single clinical research unit of Project MATCH also is a limitation that bears upon the study's generalizability. Current efforts are under way to examine these effects in several additional sites from Project MATCH. Despite these sample size limitations, there is probably much to be learned from those 'outliers' who were matched or mismatched. A prospective study of these constructs wherein patients are assigned systematically to matched or mismatched interventions would help to provide more definitive evidence. Such a study is an important next step in this line of research. However, if in subsequent work mismatched interventions are found to be associated with clinical deterioration, a prospective study that assigns patients to a mismatch condition may not be acceptable on ethical grounds.

In summary, the current work provides evidence for the negative role of patient-treatment mismatches in alcohol treatment effectiveness. Some of the patienttreatment matches studied herein showed a beneficial effect, although the magnitude of those effects were generally less than those observed for the mismatch effects. An additive effect of mismatches was also observed in which being mismatched on three or more patienttreatment pairings was associated with a particularly poor outcome. The direct analysis of matches and mismatches provided new information about the relationship between patient attributes and treatment that were not available from previous analyses of patienttreatment interactions. Future research that focuses on both replication and the use of prospective treatment designs will be important to further our understanding of these patient-treatment relationships. 


\section{Acknowledgements}

This research was supported by National Institute on Alcohol Abuse and Alcoholism (NIAAA) grant R01-AA12155.

\section{References}

1. Anton R. F., O'Malley S. S., Ciraulo D. A., Cisler R. A., Couper D., Donovan D. M. et al. Combined pharmacotherapies and behavioral interventions for alcohol dependence. JAMA 2006; 295: 2003-17.

2. Kranzler H. R., Van Kirk J. Efficacy of naltrexone and acamprosate for alcoholism treatment: a meta analysis. Alcohol Clin Exp Res 2001; 25: 1335-41.

3. Berglund M., Thelander S., Salaspuro M., Franck J., Adreasson S., Ojehagen A. Treatment of alcohol abuse. Alcohol Clin Exp Res 2003; 27: 1645-56.

4. Chick J., Anton R., Checinski K., Croop R., Drummond D. C., Farmer R. et al. A multicentre, randomized, double-blind, placebo-controlled trial of naltrexone in the treatment of alcohol dependence or abuse. Alcohol Alcohol 2000; 35: 587-93.

5. Krystal J. H., Cramer J. A., Krol W. F., Kirk G. F., Rosenheck R. A. Naltrexone in the treatment of alcohol dependence. N Engl J Med 2001; 345: 1734-9.

6. Longabaugh R., Zweben A., LoCastro J. S., Miller W. R. Origins, issues and options in the development of the combined behavioral intervention. J Stud Alcohol 2005; 15: 179-87.

7. Miller W. R., editor. Combined Behavioral Intervention: Therapist Manual. Bethesda, MD: National Institute on Alcohol Abuse and Alcoholism; 2000.

8. Kadden R., Carroll K., Donovan D., Cooney N., Monti P., Abrams D. et al. Cognitive Behavioral Coping Skills Therapy Manual: a Clinical Research Guide for Therapists Treating Individuals with Alcohol Abuse and Dependence. Project MATCH Monograph Series, vol. 3. Bethesda, MD: National Institute on Alcohol Abuse and Alcoholism; 1995.

9. Meyers R. J., Smith J. E. Clinical Guide to Alcohol Treatment: the Community Reinforcement Approach. New York: Guilford Press; 1995.

10. Nowinski J., Baker S., Carroll K. The Twelve-Step Facilitation Manual. A Clinical Research Guide for Therapists Treating Individuals with Alcohol Abuse and Dependence. Project MATCH Monograph Series, vol. 1. Bethesda, MD: National Institute on Alcohol Abuse and Alcoholism; 1995.

11. Project MATCH Research Group. Matching alcoholism treatments to clients heterogeneity: Project MATCH posttreatment drinking outcomes. J Stud Alcohol 1997; 58: 7-29.

12. Project MATCH Research Group. Project MATCH secondary a priori hypotheses. Addiction 1997; 92: 1671-98.

13. Mattson M. E., Allen J. P., Longabaugh R., Nickles C., Connors G. J., Kadden R. A chronological review of empirical studies matching alcoholic clients to treatment. J Stud Alcohol Suppl 1994; 12: 16-29.

14. Karno M. P., Longabaugh R. Patient depressive symptoms and therapist focus on emotional material: a new look at Project MATCH. J Stud Alcohol 2003; 64: 607-15.

15. Karno M. P., Longabaugh R. What do we know? Process analysis and the search for a better understanding of Project MATCH's anger by-treatment matching effect. J Stud Alcohol 2004; 65: 501-12.

16. Karno M. P., Longabaugh R. An examination of how therapist directiveness interacts with patient anger and reactance to predict alcohol use. J Stud Alcohol 2005; 66: 825-32.

17. Karno M. P., Longabaugh R. Less directiveness by therapists improves drinking outcomes of reactant clients in alcoholism treatment. J Cons Clin Psychol 2005; 73: 262-7.

18. Speilberger C. D. Manual for the State-Trait Anger Expression Scale (STAX). Odessa, FL: Psychological Assessment Resources; 1988.

19. Beck A. T., Ward C. H., Mendelsohn M., Mock J., Erbaugh J. An inventory for measuring depression. Arch Gen Psychiatry 1961; 4: 561-71.

20. Fisher D., Beutler L. E., Williams O. B. Making assessment relevant to treatment planning: the STS clinician rating form. J Clin Psychol 1999; 55: 825-42.

21. Fisher D. R., Karno M. P., Sandowicz M., Albanese A., Beutler L. E. Systematic Treatment Selection Therapy Rating Scale Manual. Santa Barbara, CA: University of California; 1995.

22. Miller W. R. Form 90: a Structured Assessment Interview for Drinking and Related Behaviors: Test Manual. NIAAA Project MATCH monograph series. 5: NIH Publication no. 96-4004. Bethesda, MD: Department of Health and Human Services; 1996.

23. Cisler R. A., Kowalchuk R. K., Saunders S. M., Zweben A., Trinh H. Q. Applying clinical significance methodology to alcoholism treatment trials: determining recovery outcomes status with individual- and population-based measures. J Alcohol Clin Exp 2005; 29: 1991-2000.

24. Jacobson N. S., Truax P. Clinical significance: a statistical approach to defining meaningful change in psychotherapy research. J Consult Clin Psychol 1991; 59: 12-19. 